\title{
Influence des conditions d'engorgement du sol sur l'évolution de l'état hydrique de jeunes plants d'Epicéa (Picea abies L)
}

\author{
A. GRANIER * et G. LÉVY * \\ avec la collaboration technique de Y. LefeVre** \\ * Station de Svlvicullure et de Production. \\ * Station de Recherches sur les Sols forestiers et la Fertilisation \\ Contre de Recherche's forcstieres de Nancy, I.N.R.A., \\ Champenoux, 54280 Seichamps
}

\begin{abstract}
Résumé
De jeunes plants d'Epicéa ont été soumis à différents types d'engorgement : nappe en surface ou à différentes profondeurs, «oxygénées» (barbotage d'air ou renouvellement quotidien de l'eau) ou non. L'état hydrique des plants a été évalué au moyen de la pression de sève, soit en conditions de transpiration nulle (pression de base), soit lorsqu'il y a transpiration.

Les principaux résultats sont les suivants :

- La pression de base des plants (mesures nocturnes) diminue au cours des premiers jours d'engorgement dans le cas d'une nappe non oxygénée de surface; après le $13^{\circ}$ jour, cette pression augmente rapidement. Lorsque le ressuyage intervient alors que la pression de base dépasse de 3 bars au moins celle des témoins, tous les plants meurent; le ressuyage accélère beaucoup l'augmentation du déficit hydrique ainsi que la mortalité de ces plants.

Pour des nappes superficielles «oxygénées» ou dans le cas d'une nappe à $-3 \mathrm{~cm}$, le déficit hydrique de base apparaît plus tardivement, ou pas du tout.

- Les déficits hydriques apparaissent plus rapidement de jour que de nuit, et en jour ensoleillé qu'en jour couvert. Pour certains traitements (nappe plus profonde, par exemple à $-6 \mathrm{~cm}$ ), le déficit n'apparaît d'ailleurs que durant la journée.

- Des apports d'engrais ont montré que la carence azotée provoquée par la nappe contribuait beaucoup à l'apparition ou à l'importance du déficit hydrique. Il en est de même, mais dans une moindre mesure, d'un déficit du sol en phosphore.
\end{abstract}

\section{I. - Introduction}

Un engorgement important du sol crée au niveau de l'enracinement des végétaux des conditions hypoxiques ou même anoxiques. Les racines ne sont plus en mesure de fonctionner normalement; il est en particulier connu que ces conditions peuvent paradoxalement entraîner un déficit dans l'alimentation en eau de ces végétaux (HELLER, 1969). 
Nous avons ainsi établi précédemment qu'un engorgement de surface provoquait en effet un déficit hydrique dans les parties aériennes de jeunes plants d'Epicéa; ce déficit contribuait à la diminution de croissance aérienne des plants et, si l'engorgement se poursuivait assez longtemps, constituait la cause directe de leur mort, au moins sur les sols hydromorphes généralement assez riches de l'Est de la France; nous l'avons constaté notamment à l'aide de nébulisation d'eau sur les feuilles (LEVY, 1981 a).

Il ne s'agissait cependant là que d'une étude qualitative et limitée. Aussi avonsnous effectué sur de jeunes plants d'Epicéa une étude dynamique quantitative de l'apparition et de l'évolution de ce déficit hydrique, à différents moments de la journée, en fonction des caractéristiques de la nappe et du sol, et de ses liaisons avec la mortalité des plants.

Nous avions également mis en évidence précédemment le rôle de l'état de la nutrition minérale des plants sur leur survie en sol engorgé : la forte carence azotée créée par la nappe, et à un degré moindre un certain déficit du sol en phosphore, contribuaient à augmenter fortement la mortalité. Nous avons supposé que cette conséquence des déficiences minérales était directement provoquée par une augmentation du déficit hydrique interne, hypothèse que nous avons tenté ici de vérifier quantitativement et de généraliser en l'étendant au cas de nappes moins néfastes, qui ne provoquent pas la mort des plants.

Il n'existe à notre connaissance dans la littérature que très peu d'informations sur l'évolution de l'état hydrique des arbres sous l'influence des conditions de l'engorgement du sol.

\section{II. - Matériel et méthodes}

Les études de potentiel hydrique ont été effectuées en serre dans le cadre de plusicurs expériences, et se sont étalées sur 3 années successives.

Nous avons utilisé des vases de végétation cylindriques d'environ $30 \mathrm{~cm}$ de diamètre et de hauteur, dans lesquels la nappe était installée au niveau désiré grâce à un dispositif décrit précédemment (LEvy, 1981 a). Le fond des vases est garni de gravillons sur une hauteur de $5 \mathrm{~cm}$. Deux types de sols ont été choisis : le sol «Amance » qui est un mull mésotrophe à texture limoneuse $(\mathrm{C} / \mathrm{N}=14, \mathrm{~S} / \mathrm{T}=61$, $\mathrm{P}_{2} \mathrm{O}_{5}$, assimilable $=0,11$ p. 1000 ), représentatif de très nombreux sols à hydromorphie temporaire de l'Est de la France, utilisé pur ou en couche d'environ $7 \mathrm{~cm}$ d'épaisseur surmontant un sable grossier, et le sol «Mondon», qui est un mull acide à texture limono-sableuse, plus pauvre en $\mathrm{P}_{.2} \mathrm{O}_{5}(0,07$ p. 1000$)$, tous deux prélevés dans des stations hydromorphes.

Nous avons repiqué dans chaque vase 9 plants d'Epicéa âgés de 2 ans, et établi la nappe la même année, après le temps nécessaire à leur installation, très souvent alors que la pousse terminale de l'année avait une longueur d'environ $1 \mathrm{~cm}$, parfois plus tardivement. 
Dans certains cas, nous avons amélioré le potentiel d'oxydo-réduction du milicu. soit par insufflation permanente d'air à partir du fond des vases à l'aide de pompes d'aquarium, soit par renouvellement quotidien de la nappe (nappes "oxygénées»). Le potentiel redox sabaisse dans tous les traitements quand la duréc de l'engorgement augmente, mais il reste bien sûr constamment plus élevé pour les nappes «oxygénées».

Des apports d'éléments nutritifs ont été effectués sur le sol à deux reprises, à 7 à 10 jours d'intervalle, peu avant l'engorgement. Juste avant celui-ci, nous avons «lavé » les sols plusieurs fois afin d'éliminer les sels solubles qui auraient augmenté fortement la pression osmotique de la nappe. Les doses suivantes ont été appliquées à chacune des deux reprises :

$\mathrm{N}: 3 \mathrm{~g}$ d'ammonitrates à 34 p. 100 d'azote,

$\mathrm{PK}$ : $50 \mathrm{ml}$ de solution de $\mathrm{PO}_{4} \mathrm{H}_{2} \mathrm{~K}$ à $40 \mathrm{~g} / \mathrm{l}$,

P : $50 \mathrm{ml}$ de solution de $\mathrm{Ca}\left(\mathrm{PO}_{4} \mathrm{H}_{22}\right)_{2}, \mathrm{H}_{2} \mathrm{O}$, à $40 \mathrm{~g} / \mathrm{I}$,

$\mathrm{K}$ : $50 \mathrm{ml}$ de solution de $\mathrm{SO}_{4} \mathrm{~K}_{2}$ à $30 \mathrm{~g} / 1$.

L'influence de la nappe a toujours été indiquée par comparaison avec un témoin non engorgé dont le sol est maintenu à la capacité au champ.

Les vases de végétations ont été disposés sur de grandes tablettes, par blocs.

\section{Mesure du potentiel hydrique}

L'état hydrique des plants d'Epicéa au niveau des ramcaux a été caractérisé par le potentiel hydrique. Nous avons utilisé la méthode de la chambre à pression (Scholander et al., 1965). Selon Ritchie \& Hinckley (1975), nous pouvons décomposer le potentiel hydrique de la sève $(\Psi)$ en plusieurs facteurs :

$$
\Psi=-\Psi_{\mathrm{g}}-\Psi_{\mathrm{T} \mathbf{R}}-\pi-\tau-\Psi_{\mathrm{s}}
$$

où $\Psi_{\mathrm{g}}$, représente le potentiel de gravité $(0,1 \mathrm{bar} / \mathrm{m})$, I $\Psi_{\mathrm{T}}$ le le potenticl de friction (dû au passage du flux de transpiration), $\pi$ et $\tau$ les potenticls osmotique ct matriciel, et $\Psi_{\mathrm{s}}$ le potentiel de l'eau dans le sol.

La chambre à pression permet de mesurer le terme $\mathbf{P}$ de l'équation (2) :

$$
\Psi=\mathbf{P}-\pi
$$

(Boyer, 1967) où $\mathrm{P}$ désigne la pression d'extraction de la sève; or, pour la sève brute on peut considérer $\pi \simeq \mathrm{O}$ (BOYER, 1969), et dans ces conditions $\Psi \simeq \mathrm{P}$. La méthode psychrométrique (BARRs, 1965; Brown, 1976, etc.), qui permet de mesurer directement le potentiel hydrique de la feuille (et non de la sève brute), peut être considérée comme une méthode de référence. Divers auteurs ont comparé les mesures effectuées au moyen de la chambre à pression avec celles fournies par la technique psychrométrique; dans la plupart des cas la relation est linéaire, pour les résineux tout au moins; néanmoins, les valeurs obtenues par les deux méthodes ne sont pas absolument identiques; l'écart dépend des espèces (voir en particulier BoYER, 1967 et 1969 ; KaufmanN, 1968 ; Ritchie \& Hinckley, 1971; Barker, 1973; Talbot et al., 1975). 
La chambre à pression qui donne donc des mesures estimant correctement létat hydrique du rameau est en plus un instrument d'emploi plus commode et plus rapide que la chambre psychrométrique.

L.e gradient de potentiel hydrique dans un organe végétal détermine le mouvement de l'eau (sève brute) vers le potentiel le plus faible. Cette convention des physiciens implique que le potentiel dans le rameau est toujours négatif, celui d'une nappe deau libre étant nul. Nous appellerons pression de sève la valeur lue sur le manomètre de la chambre à pression; elle est donc positive, égale et opposée au potentie! de sève.

\section{Signification de la pression de base $P_{B}$}

La mesure de la pression de sève en fin de nuit représente un point caractéristique dans la cinétique d'évolution du potentiel hydrique (HINCKLEY \& RITCHIE, 1973 ; Aussenac \& Granier, 1978). Ce point est appelé pression de base ; c'est la valeur vers laquelle tend la pression de sève en l'absence de transpiration (donc durant la nuit); il y a alors équilibre hydrique dans le végétal. Selon Hinckley et Ritchie, 1973, la pression de base dépend principalement :

- du potentiel de l'eau dans le sol,

- de la capacité d'absorption des racines.

Les mesures de pression de base ont été faites en fin de nuit, ou à défaut après le lever du soleil, mais sur des plants préalablement recouverts d'un cache noir et étanche supprimant toute transpiration. Une expérience préliminairc a permis de vérifier la validité de cette façon d'opérer.

Signification de la pression de sève dans la journée

Selon Hinckley \& Ritchie, 1973, la pression de sève $P(t)$ au temps $t$ est donnée par la relation:

$$
\mathrm{P}(\mathrm{t})=\mathrm{P}_{\mathrm{I}}+\Delta \mathrm{P}
$$

où $P_{\mathrm{I}}$ représente la pression de base et $\Delta P$ la variation induite par les conditions extérieures (évapotranspiration potentielle) et internes (mécanismes de régulation).

Ainsi, tous autres facteurs égaux par ailleurs, l'augmentation de l'E.T.P. induira un accroissement de $\Delta \mathbf{P}$ et donc de $\mathbf{P}(\mathrm{t})$.

Notons que toutes les mesures ont porté sur des plants en bon état physiologique, tout au moins en apparence.

Signalons enfin que deux mesures ont été considérées comme différentes si leur écart dépassait 1 bar. 


\section{III. - Résultats}

\section{1. - Pression de base}

\subsection{Evolution dans le temps}

a) Nappe en surface

La figure 1 représente l'évolution de la pression de base en fonction de la durée d'engorgement pour une nappe stagnante non «oxygénée», chaque point étant la moyenne de plusieurs mesures (au moins trois). Les résultats obtenus sur les deux types de substrats (《Mondon» et «Amance») y ont été rassemblés, car les deux catégories de plants présentent le même comportement :

- entre le $2^{\circ}$ et le $13^{\prime \prime}$ jour après l'engorgement, on note une diminution de la pression de sève par rapport au témoin; elle passe de 7 bars à 3 bars;

- après le 13" jour, la pression augmente rapidement;

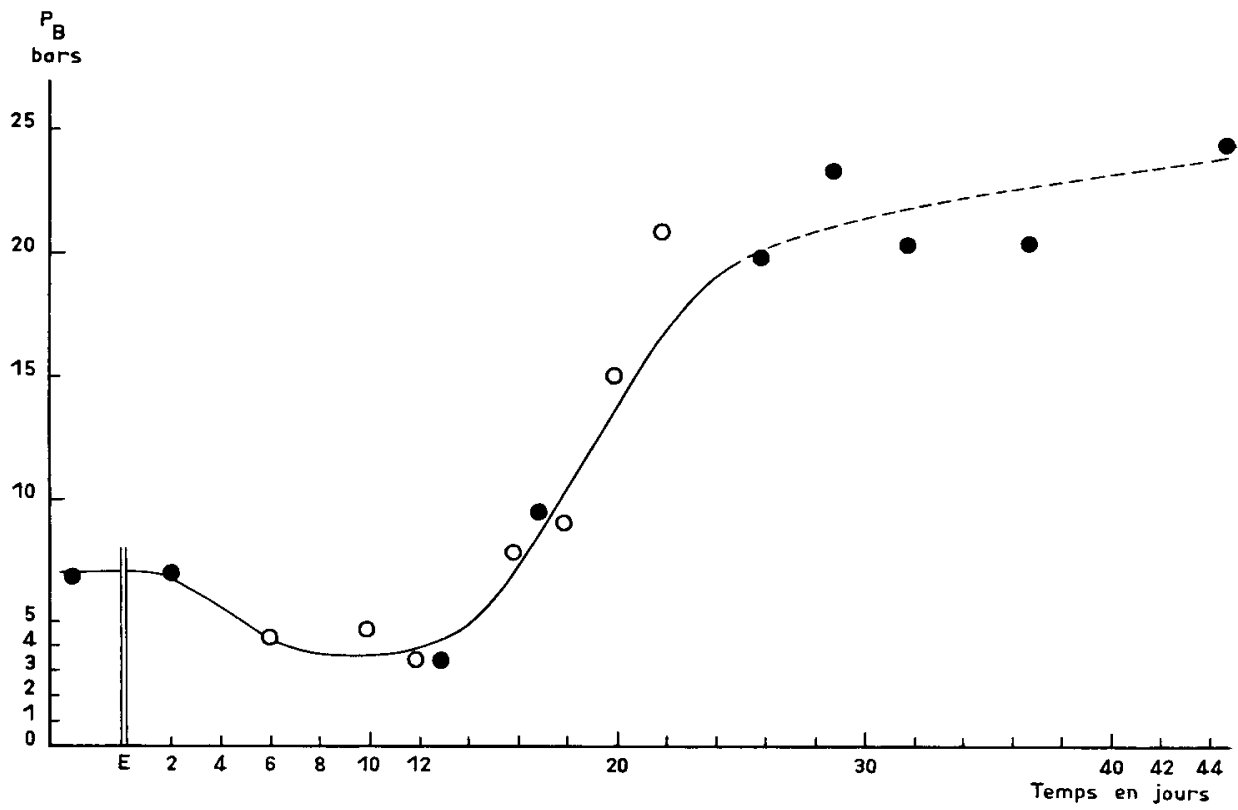

- Sol AMANCE

- Sol MONDON

FIG. 1

Evolution de la pression de base $\left(P_{B}\right)$ en fonction du temps après le début de l'engorgement $(E)$ en conditions de nappe stagnante superficielle

Evolution of basic pressure $\left(P_{B}\right)$ as a function of time after beginning of water logging $(E)$ in stagnant surface water-table conditions 
- après le $26^{\mathrm{e}}$ jour environ, la pente de la courbe diminue sensiblement. Mais il faut signaler que l'appareil ne permettait pas de mesurer des pressions supérieures à 30 bars; les pressions supérieures à cette valeur étant notées 30 bars, les moyennes ont ainsi été sous-estimées (la partie correspondlante de la courbe a été représentée en traits pointillés).

Il est nécessaire pour les autres traitements où la nappe est en surface (barbotage d'air, renouvellement quotidien ou apport de nitrates) de dissocier les deux types de substrats, où des évolutions différentes de la pression ont été relevées.

\section{- Sol «Amance» (fig. 2)}

Dans le traitement avec barbotage d'air, la pression de base augmentait lentement dans le temps, sans toutefois dépasser la valeur de 12 bars, soit 5 bars de plus que pour le témoin non engorgé.

Au contraire, dans le traitement où l'eau est renouvelée tous les jours, les pressions de base restent identiques à celles des témoins. Enfin, dans le traitement avec apports de nitrates, la pression de sève augmente tout au long de l'expérience, mais de façon moins rapide que pour le traitement où la nappe est stagnante.

\section{- Sol « Mondon»}

Les différents traitements où intervient une oxygénation du substrat sont plus défavorables sur le sol «Mondon» que sur sol «Amance», comme l'attestent les valeurs élevées des pressions de sève sur le tableau 1 :

\section{TABleau 1}

Pression de base (moyennes) sur sol «Mondon» après différentes durées d'engorgement

\begin{tabular}{|c|c|c|c|c|c|}
\hline \multirow{2}{*}{ Traitement } & \multicolumn{5}{|c|}{ Jours d'engorgement } \\
\hline & 18 & 20 & 22 & 24 & 28 \\
\hline Barbotage d'air . .............. & 11,9 & 13,3 & & 23,6 & \\
\hline Renouvel'ement quotidien $\ldots \ldots \ldots \ldots$ & & & 11,5 & 14,5 & \\
\hline Nappe à $-3 \mathrm{~cm} \ldots \ldots \ldots \ldots$ & & & 12,2 & 8,0 & 11,2 \\
\hline
\end{tabular}

Témoin non engorgé $=7,0$ bars.

Ces valeurs n'ont pas été reportées sur la figure 2 afin de ne pas nuire à sa clarté, car elles concernent une période de temps très réduite par rapport aux traitements correspondants sur sol «Amance».

Pour les deux sols, le traitement à renouvellement quotidien de l'eau conduit à des pressions plus faibles que celui qui comporte un barbotage d'air. Toutefois ces deux traitements provoquent un déficit hydrique plus prononcé sur sol «Mondon» que sur sol «Amance». 


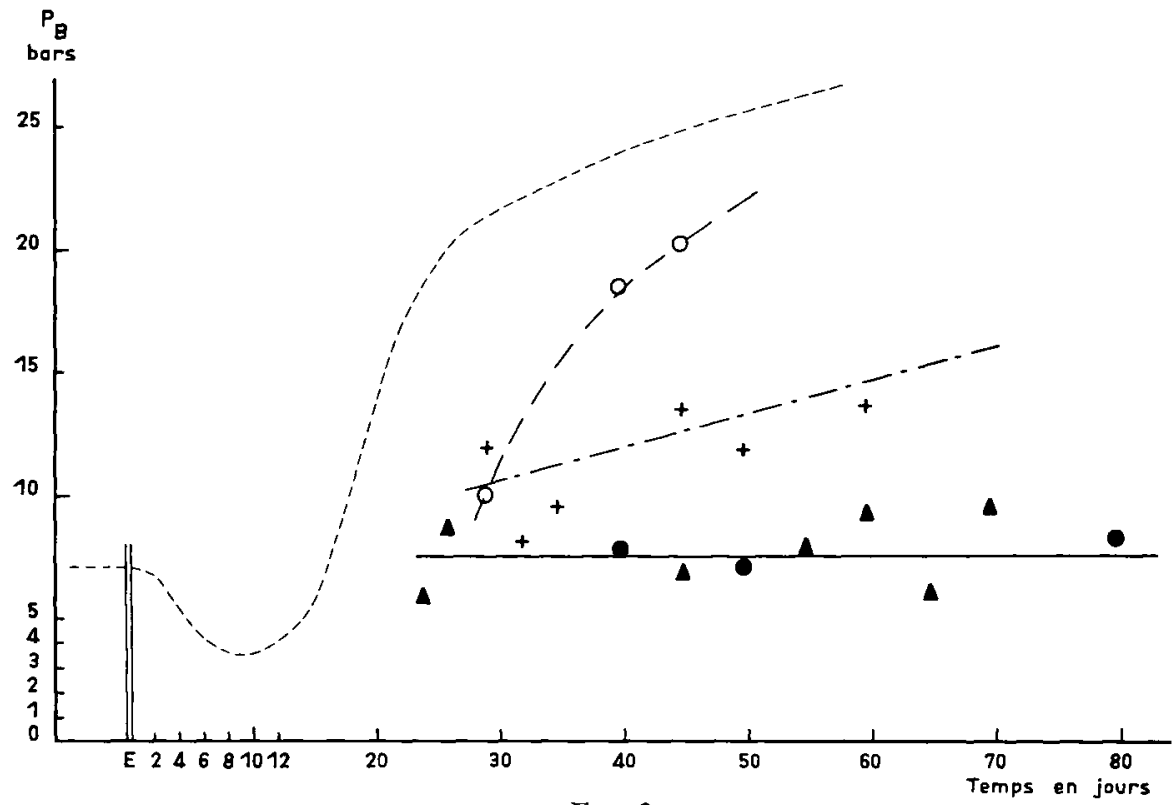

FIG. 2

Evolution de la pression de base $\left(P_{k}\right)$ en fonction du temps après le début de l'engorgement (E) pour différents traitements

Evolution of basic pressure $\left(P_{B}\right)$ as a function of time after beginning of water logging $(E)$

Nappe en surface for different conditions

Surface water-table

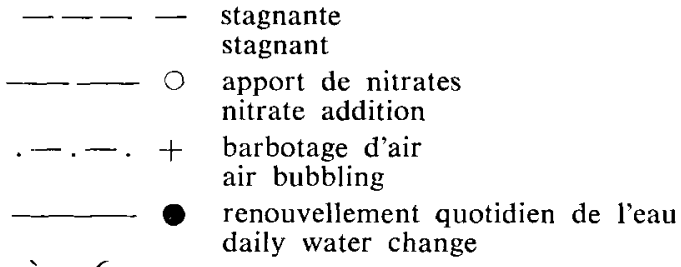

Nappe $\grave{a}-6 \mathrm{~cm}$

$6 \mathrm{~cm}$ deep water-table

b) Nappe à différents niveaux

Deux profondeurs de nappe ont été envisagées : l'une à $-3 \mathrm{~cm}$, l'autre à $-6 \mathrm{~cm}$. Nous appellerons les traitements correspondants -3 et -6 . Les plants du traitement - 6 ont réagi de la même façon sur sol "Amance» que sur sol «Mondon». La pression de base reste en moyenne à 7 bars, qui est la valeur du témoin non engorgé. Le traitement - 3 , sur sol «Mondon» uniquement, est à un niveau de 11,2 bars au bout de 28 jours d'expérience (voir tableau 1). Ce traitement n'a pas été étudié sur sol de type «Amance». 
Tous ces traitements étaient suivis d'un ressuyage total, effectué en une seule fois, après différentes durées d'engorgement ; les sols étaient maintenus ensuite à la capacité au champ.

\subsection{2. - Liaison entre le potentiel de base au moment du ressuyage et la mortalité des plants}

Notons qu'aucun ressuyage n'a été effectué avant le 14" jour d'engorgement sur sol «Amance». Une durée d'engorgement inférieure ne provoque pratiquement aucune mortalité, nous l'avons constaté par ailleurs.

\section{a) Sol «Amance»}

La figure 3 permet de comparer la mortalité des plants en fonction de leur pression de base au moment du ressuyage; sur cette figure, les pressions ont été regroupées par classes. Les observations de mortalité ont été effectuées au début de l'année suivante.

Nous constatons sur le diagramme de cette figure 3 que :

-. tous les plants à pression de base supérieure à 10 bars au moment du ressuyage sont alors morts ou destinés à mourir ;

- 50 p. 100 des plants survivent si leur pression au ressuyage est comprise entre 7,5 et 10 bars, et 88 p. 100 si elle se situe entre 5 et 7,5 bars;

- les plants qui présentent une pression inférieure à 5 bars lors de leur ressuyage ( $1^{\text {r" }}$ colonne du diagramme) sont tous destinés à mourir. Sur la figure 1, ces plants sont pris en compte dans les moyennes représentées au-delà de 16 jours d’engorgement. Mais il s’agit là d'une cause de mortalité supplémentaire autre que le déficit hydrique des rameaux (probablement de nature pathologique). Les plants de cette catégorie présentaient des aiguilles à extrémité rosissantes ou rouges, leur pression de base restant toujours proche de zéro.

Il est donc déjà possible de donner, pour les plants laissés en nappe stagnante, le schéma suivant :

I.es plants voient leur potentiel évoluer en moyenne selon la cinétique de la figure 1 :

- le niveau 10 bars est atteint, en moyenne, au 18" jour d'engorgement;

- Ia mortalité est totale au-dessus de 10 bars au moment du ressuyage, donc dans les conditions de notre expérience après 18 jours d'engorgement.

Précisons quà partir du moment où ce niveau 10 bars est atteint, les plants sont destinés à mourir, que l'on ressuie ou non le sol.

Mais il faut signaler que cette valeur limite de 18 jours est une moyenne et qu un petit nombre de plants n'atteignent les 10 bars qu'au bout de 26 jours d'engorgement. 


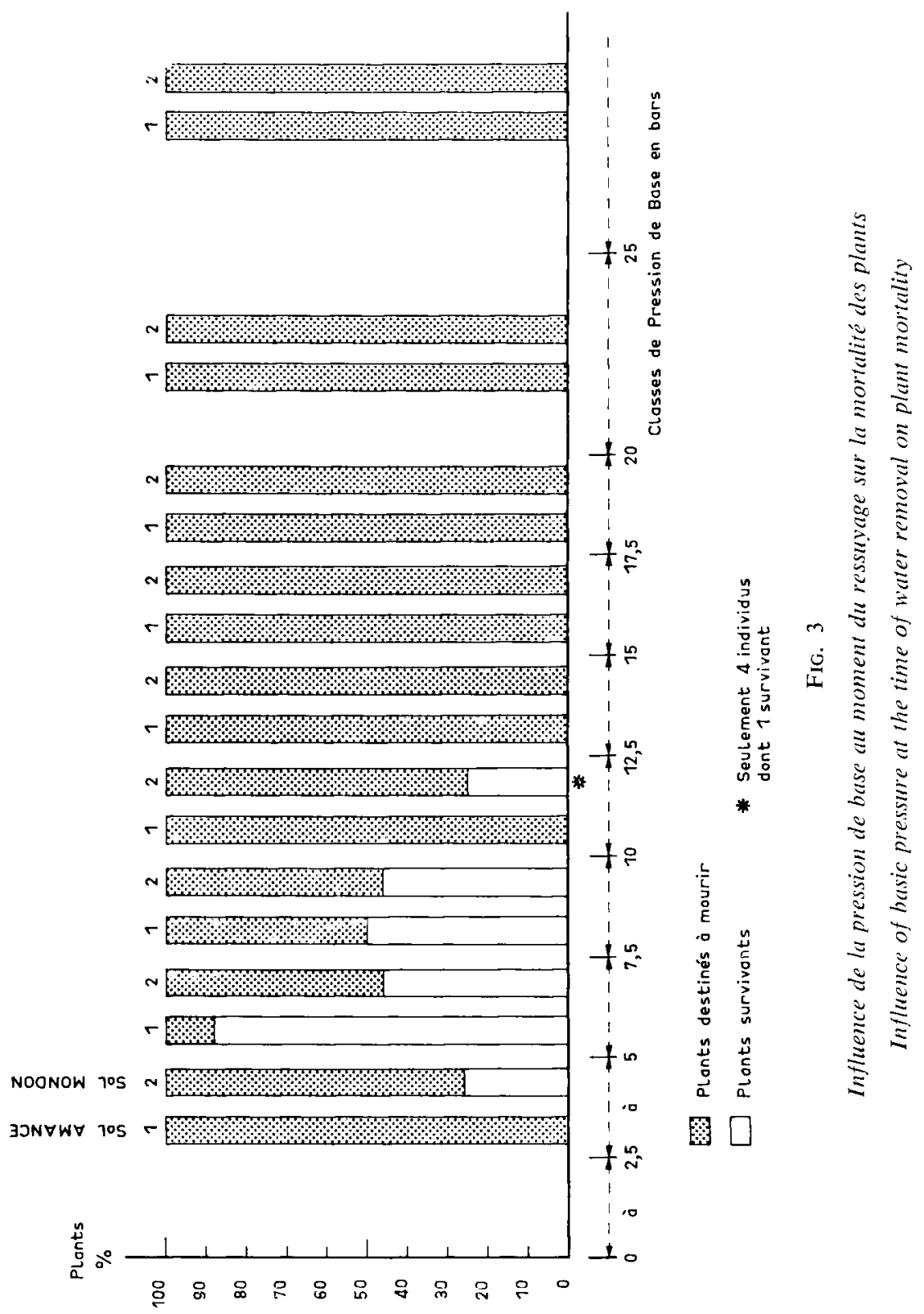


b) Sol «Mondon»

Dans l'ensemble, les plants repiqués sur sol de type «Mondon» réagissent comme ceux sur sol «Amance».

Trois remarques s"imposent sur la comparaison des sols «Amance» et «Mondon $\gg$ (fig. 3) :

— sur sol «Mondon», les 100 p. 100 de mortalité ne semblent atteints qu'audessus de la pression 12,5 bars au ressuyage. Mais il est à noter que les 25 p. 100 indiqués figure 3 dans la classe de 10 à 12,5 bars ne correspondent quà un survivant, sur un lot de seulement 4 plants;

- au-dessous de 5 bars au ressuyage, on constate que la mortalité est élevée $(75$ p. 100$)$, mais elle n'atteint pas 100 p. 100 . De la même façon que sur sol «Amance», cette classe comprend les plants en mauvais état sanitaire (extrémités rosissantes), tous destinés à mourir. Les 25 p. 100 qui survivent sont en fait des individus ressuyés très tôt, dans la première phase de la figure 1 , entre le 3 " et le $10^{\prime \prime}$ jour ;

- la classe des 5 et 10 bars au moment du ressuyage est celle qui contient le plus de survivants, en plus faible taux que sur sol «Amance» : 47 p. 100 contre 50 à 88 p. 100 . Nous avons en effet remarqué que dans cette classe, les manifestations extérieures du dépérissement dorigine sans doute pathologique étaicnt bien plus fréquentes sur sol «Mondon» que sur sol «Amance».

\subsection{3. - Influence du ressuyage sur l'évolution de la pression de base}

La figure 4 reporte l'évolution moyenne, après ressuyage, de la pression de base de plants sur sol de type «Amance». Les plants ont été regroupés par classes de pression de base au moment du ressuyage, «calées» ensuite sur la courbe moyenne de la figure 1. Il est ainsi possible de comparer l'évolution de la pression de base de plants ressuyés à celle des plants restant en nappe stagnante. On peut distinguer deux catégories de plants :

- les individus dont la pression est supérieure à 10 bars au moment du ressuyage. Cette pression augmente très vite, beaucoup plus que sills étaient restés engorgés. Rappelons que tous ces plants sont destinés à mourir (fig. 3) ;

- les individus de la classe 5 à 10 bars atı moment du ressuyage voient ensuite leur pression de sève augmenter légèrement, mais de façon moindre que les plants laissés en nappe stagnante. Cette augmentation est faible (3 bars au maximum) et de courte durée, puisque la pression de base diminue dans les dix jours qui suivent le ressuyage, pour atteindre la valeur de 7 bars, celle des témoins non engorgés. Les points représentés sur le graphique ne concernent en fait que des plants survivants (qui constituent environ 70 p. 100 de la classe).

Remarque: Sur la figure 4, nous n'avons pas représenté les plants restés à une pression faible longtemps après le début de l'engorgement. Ces plants en mauvais état sanitaire voient, après ressuyage, leur pression augmenter très fortement en quelques jours.

Donc, pour les plants destinés à survivre, le ressuyage abaisse au bout de quelques jours leur pression de base par rapport à ceux maintenus engorgés. Au contraire, pour les plants destinés à mourir, le ressuyage augmente considérablement la pression de base par rapport aux engorgés. 


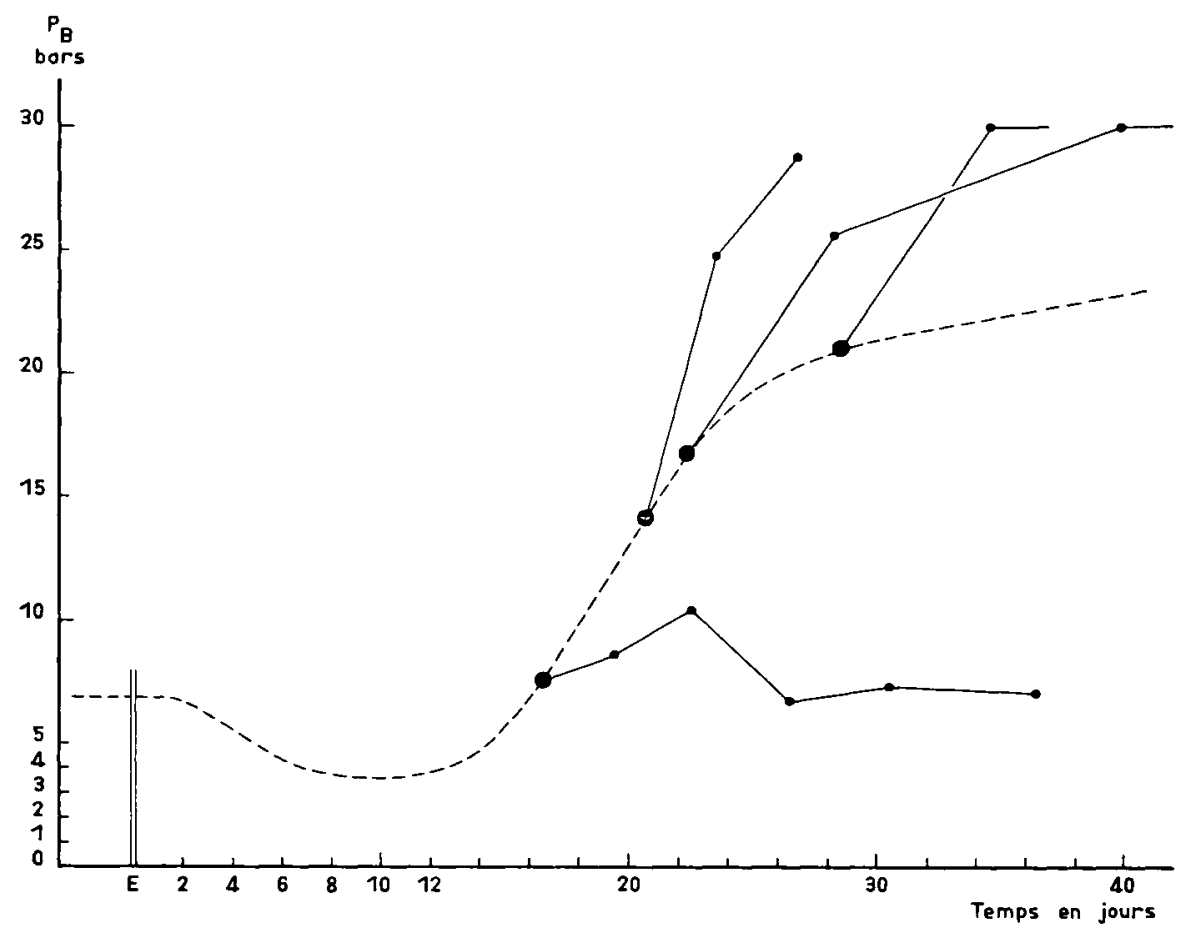

FiG. 4

Evolution de la pression de base $\left(P_{B}\right)$ après ressuyage (- - ), comparée à celle de plants demeurés en nappe stagnante superficielle (- . - -)

Evolution of basic pressure $\left(P_{B}\right)$ after water-table removal (- - ). compared to the one of plants left in stagnant surface water-table (--.)

Nous avons considéré jusqu'ici la pression de base, c'est-à-dire létat d'équilibre entre les plants et le sol lorsque la transpiration est nulle. Voyons maintenant ce qui diffère, pour ces mêmes traitements, dans les mesures de pression de sève de jour.

\section{2. - La pression de sève au cours de la journée}

\subsection{1. - Les plants témoins (sol à la capacité au champ)}

Nous avons effectué des mesures de pression ce sève sur le témoin tout au long d'une même journée ensoleillée (sol «Amance») (fig. 5). On constate que la valeur maximum constitue un palier, qui débute vers $8 \mathrm{~h}$ (peu après le moment où la serre commence à être ensoleillée) et se maintient au moins jusque vers 17 h 30 .

Nos mesures de jour ont été bien entendu toujours effectuées dans cet intervalle, en général entre $11 \mathrm{~h} 30$ et $16 \mathrm{~h}$. 


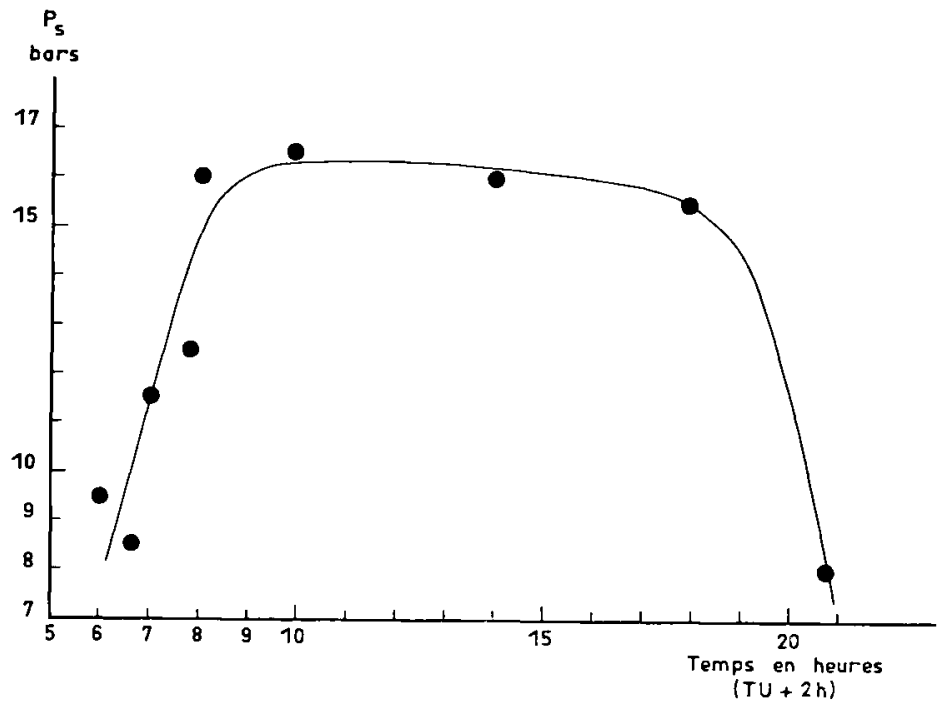

FIG. 5

Evolution de la pression de sève (Ps) dés plants témoins (sol à la capactié au champ) au cours d'une journée cusolcillíe

Evolution of sap pressure (Ps) of plants growing on soil at field capacity during a sunny day

La valeur du palier au cours d'une journée donnée dépend évidemment des conditions climatiques correspondantes.

Bien que chacun des deux termes qui suivent recouvre en réalité des conditions assez hétérogènes, nous avons, par commodité, séparé les mesures en jours couverts et celles en jours ensoleillés; c'est en effet la présence ou l'absence de soleil qui est le facteur essentiel de la transpiration, responsable de l'accroissement de pression $\Delta \mathrm{P}$ [formule (3)].

Il apparaît cependant que les conditions climatiques du (ou des) jour précédent ont une importance certaine. Ainsi, au cours d'une même expérience, nous avons relevé les valeurs suivantes de pression de sève (en bars) pour le témoin dans la journée, pour le sol "Amance», au cours dune période de temps relativement. courte :

— temps pluvieux depuis 2 jours (forte hygrométrie) : 7,0;

- $1^{\mathrm{rr}}$ jour de soleil après une période pluvieuse : 9,5;

- ciel couvert, depuis au moins un jour : 11,0 à 13,0;

— temps ensoleillé depuis au moins un jour : 14,0 à 16,0 .

Aussi avons-nous simplifié les résultats en considérant n'avoir affaire à un jour ensoleillé que si cette caractéristique existait déjà la veille du jour de mesure, alors que ce que nous dénommerons « jour couvert» regroupera des conditions climatiques 
plus hétérogènes. Certains résultats sont incomplets car ces conditions extérieures ne sont pas sous notre dépendance.

\subsection{2. - Nappe en surface}

La figure 6 représente (pour le sol «Mondon») l'évolution avec le temps du nombre de plants engorgés dont la pression de sève est supérieure à celle du témoin, et cela pour 3 types de conditions extérieures. On constate que, quelles que soient ces conditions, l'évolution est très rapide à partir du moment où le phénomène débute; il se manifeste plus précocement de jour que de nuit (pression de base) et, dans la journée, si le temps est ensoleillé que si le ciel est couvert. Les conditions climatiques n'ont pas permis de prolonger la courbe «jour ensoleillé » att-delà du $9^{r}$ jour d'engorgement.

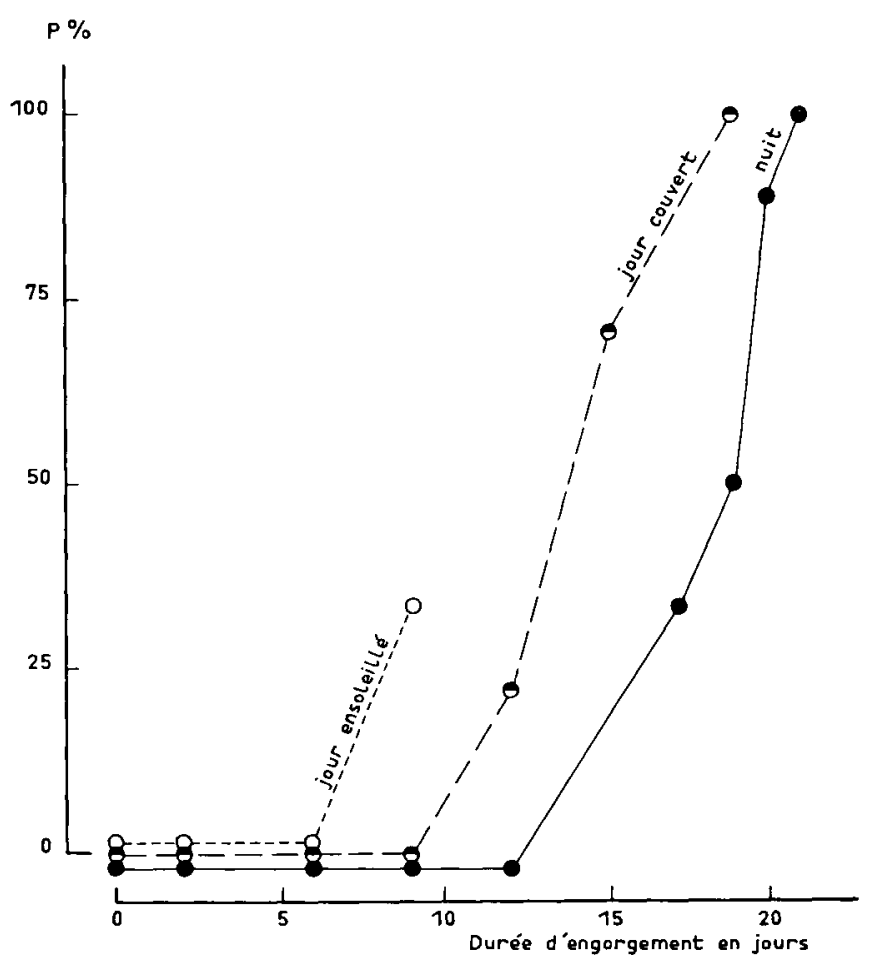

FIG. 6

Evolution du pourcentage de plants $(P \%)$ à pression de sève supérieure au témoin, con fonction du temps, lors de mesures diurnes et nocturnes

Evolution of plants percentage $(P \%)$ with sap pressure above the one of plants growing on soil at field capacity, as a function of time, for day and night measurements 
L'augmentation moyenne de pression des plants concernés, par rapport au témoin, est également rapide. Ainsi, en jours couverts, elle est dans cette même expérience de 5 bars du $12^{\circ}$ au $15^{\circ}$ jour de l'engorgement, de 6,5 bars au $16^{\circ}$ jour; au $19^{\circ}$ jour tous les plants sont concernés par cette augmentation, qui est au minimum de 11 bars, alors que la grande majorité des plants a une pression supérieure à 30 bars (au moins 18 bars de plus que le témoin) : on pcut supposer que cette très forte augmentation entre le $16^{\circ}$ et $19^{\prime \prime}$ jour est en rapport avec la mortalité des plants.

Avant que la pression ne commence à augmenter par rapport au témoin, elle lui est très souvent inférieure au cours de la journée, surtout en jour ensoleillé (il en est de même, nous lavons signalé, durant la nuit).

La pression de sève de pratiquement tous les plants augmente aussi par rapport au témoin, tant en jour couvert qu'en jour ensoleillé, si cette nappe superficielle est oxygénée, soit par barbotage d'air, soit par renouvellement quotidien de l'eau. Cette augmentation apparaît cependant plus tardivement que dans le cas d'une nappe non oxygénée. L'accroissement du nombre de plants affectés est également très rapide.

Cette augmentation générale de la pression de sève de jour et la vitesse de son évolution affectent également des plants soumis à une nappe très proche de la surface $(-3 \mathrm{~cm})$.

Pour l'ensemble de ces traitements, un certain nombre de plants, parmi ceux destinés à mourir, ont, lors du ressuyage, une pression de sève relativement faible en jour couvert (il s'agit dans leur cas de la mortalité d'origine probablement pathologique que nous avons signalée précédemment). De toute façon, tous les plants dont la pression de sève atteint ou dépasse 25 bars en jour couvert lors du ressuyage meurent ultérieurement.

\subsection{3. - Nappe plus profonde}

Lorsque la nappe se situe à $-6 \mathrm{~cm}$, l'évolution de la pression de sève est très différente de ce qu’elle était pour des nappes plus superficielles.

En jours couverts, la pression de sève d'un certain nombre de plants devient supérieure à celle du témoin à partir du $25^{\prime \prime}$ jour d'engorgement ou un peu plus tard. Il y a d'assez nettes différences entre blocs quant au nombre de plants concernés, qui varie globalement entre 10 et 50 p. 100 de l'ensemble des plants; cette valeur ne semble plus évoluer par la suite. La différence entre la pression de ces plants et celle du témoin est souvent faible ( 1 à 3 bars), elle ne dépasse d'ailleurs jamais 6 bars.

Après une même durée d'engorgement à $-6 \mathrm{~cm}$, les plants ayant une pression supérieure à celle du témoin sont plus nombreux en jours ensoleillé qu'en jour couvert (il en était de même dans le cas d'une nappe superficielle). Selon les expériences ou les blocs, cela concerne en jour ensoleillé de 30 à 60 p. 100 des plants vers le $40^{\circ}$ jour d'engorgement, valeur qui atteint 70 ou 75 p. 100 , en moyenne, aux alentours du $50^{\prime \prime}$ jour d'engorgement, et semble ensuite se stabiliser. La différence de pression par rapport au témoin ne dépasse jamais 7 bars. 
Lapparition de plants qui commencent à avoir une pression supéricure à celle du témoin, en jour couvert, est d'autant plus précoce que la nappe est plus défavorable ; ainsi, sur sol de "Mondon», environ 25 p. 100 des plants sont concernés vers le 13" jour d'engorgement pour une nappe superficielle, le $16^{\prime \prime}$ jour si celle-ci est renouvelée quotidiennement, le $19^{\circ}$ jour pour une nappe à $-3 \mathrm{~cm}$ et le $25^{\circ}$ jour, ou plus tard, si la nappe est située à $-6 \mathrm{~cm}$.

Malgré le faible nombre de mesures effectuées en jour ensoleillé, il semble que l'ordre des traitements soit le même qu'en jour couvert.

Pour des nappes maintenues à $-10 \mathrm{~cm}$, ou plus profondément, la pression de sève est toujours à peu près identique à celle du témoin au cours des journées couvertes. En jour ensoleillé, elle lui est égale ou inféricure (la différente est dans ce dernier cas de 2,5 bars en moyenne).

\section{3. - Influence de la mutrition minćrale des plants sur la pression de sève}

En raison de l'incidence pratique (par exemple sur la croissance aérienne des plants) de déficits hydriques qui ne se manifestent quau cours de la journée, donc sans entraîner la mort, nous avons testé de jour linfluence dapports de différents éléments minéraux sur la pression de sève : un plus grand éventail de conditions hydriques est concerné que si nous avions opéré de nuit.

\subsection{1. - Nappe superficielle}

Pour les deux types de sols étudiés, une fertilisation totale NPK retarde lapparition et l'évolution de l'augmentation de la pression de sève par rapport au témoin.

Des apports d'un ou deux éléments nutritifs seulement n’ont été effectués, pour ce niveau de nappe, que sur le sol de «Mondon». Les résultats sont nets; par exemple :

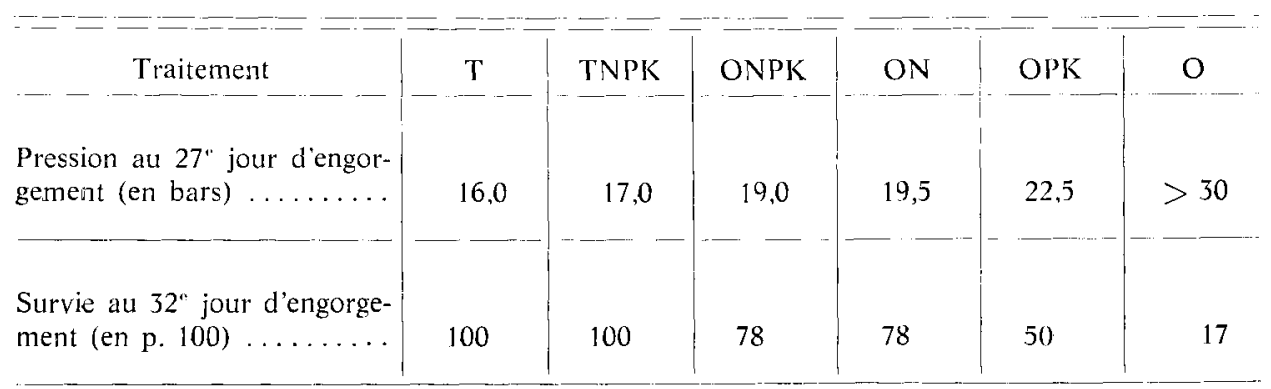

( $T=$ témoin non engorgé $; O=$ nappe en surface). 
Il existe donc une très bonne liaison entre l'augmentation de pression de sève et la mortalité. Un apport d'azote est presque aussi efficace qu'une fertilisation totale pour combattre le déficit hydrique, alors que le phosphore a aussi un certain effet positif, mais moindre. C'est cet élément qui est vraisemblablement efficace dans la combinaison PK ; en effet, dans une autre expérience avec engorgement sur le même type de sol, un apport préalable de $P$ a eu un effet positif important sur le comportement des plants, contrairement à un apport de $\mathbf{K}$.

\subsection{2. - Nappe à $-6 \mathrm{~cm}$}

Nous navons effectué des mesures, pour cette profondeur, qu'en jours ensoleillés, car ce n'est que dans ces conditions que la grande majorité des plants non fertilisés présentent un déficit hydrique par rapport au témoin.

Pour un même substrat, on obtient des résultats qualitativement semblables aux différentes dates de mesure. Par exemple, on a relevé au $74^{\prime \prime}$ jour d'engorgement :

- sur sol «Amance»:

\begin{tabular}{|c|c|c|c|c|c|c|}
\hline Traitement & $-6 \mathrm{~N}$ & $-6 \mathrm{NPK}$ & $\mathrm{T}$ & -6 & $-6 \mathrm{~K}$ & $-6 \mathrm{P}$ \\
\hline Pression de sève (en bars) & 11,5 & 11,7 & 13,3 & 15,8 & 16,2 & 19,3 \\
\hline
\end{tabular}

- et sur sol «ondon»:

\begin{tabular}{c|c|c|c|c}
\hline & Traitement \\
\hline Pression de sève (en bars) $\ldots \ldots \ldots$ & $-6 \mathrm{~N}$ & $-6 \mathrm{NPK}$ & $-6 \mathrm{P}$ & -6 \\
\hline $\mathrm{T}=$ témoin non engorgé $;-6=$ nappe à $-6 \mathrm{~cm})$.
\end{tabular}

En présence d'une nappe à $-6 \mathrm{~cm}$, la fertilisation permet donc d'abaisser la pression de sève, parfois même jusqu'au niveau de celle du témoin ou en dessous.

Lazote est aussi efficace qu'une fertilisation complète pour les deux sols utilisés (résultat comparable obtenu avec une nappe superficielle, au moins pour le sol «Mondon »).

En sol «Mondon», tout comme dans le cas d'une nappe superficielle, un apport de phosphore est utile, mais moins qu'une fertilisation azotée. Par contre en sol «Amance», un apport de phosphore est néfaste : la pression de sève devient supérieure à celle des plants non fertilisés. 


\section{IV. - Discussion et conclusion}

Lors des mesures nocturnes, en condition de transpiration nulle ou très faible, on constate, pour les plants engorgés, une augmentation de la pression de base, alors que le potentiel de leau dans le sol est très proche de zéro. Il y a done déficit d'alimentation en eau dû à une rupture de l'équilibre entre le potentiel de l'eau dans le sol et son potentiel dans le rameau.

Ce déficit dalimentation en eau en soi engorgé est logique. En effet, on sait quen hypoxie ou anoxie l'énergie fournie à la racine par le métabolisme est bien moindre qu'en normoxie. Or, l'absorption et le transfert de l'eau au niveau racinaire est un phénomène en partie actif, c'est-à-dire qui nécessite une certaine dépense d'énergie; ainsi en est-il par exemple du passage de l'cau du cortex à l'endoderme puis aux vaisseaux (MAZLIAK, 1974).

Pendant les quelques jours qui suivent lengorgement, même en surface, l'alimentation en eau est très bonne, même meilieure que celle du témoin : les racines en bon état physiologique absorbent encore mieux l'eau à un potentiel proche de zéro que l'eau dun sol à la capacité au champ.

\section{1. - Déficit hyslrique cal cours de la nuit}

Pour une nappe stagnante, ce déficit apparaît après un certain temps dengorgement (après le $13^{\circ}$ jour dans nos expériences); son aggravation est ensuite très rapide : le fonctionnement des racines est cone de plus en plus affecté.

Pour des nappes superficielles «oxygénées», ou dans le cas d'une nappe à - $3 \mathrm{~cm}$, le déficit hydrique apparaît plus tardivement, ou même jamais dans certains cas, et son évolution est beaucoup plus lente.

La mortalité des plants est très liée à cette cinétique du déficit hydrique, et en particulier à la pression de base lors du ressuyage. Il s'agit d'une liaison de causalité comme nous l'avons signalé dans l'introduction. Ainsi, lorsque le déficit de nuit au moment du ressuyage, par rapport au témoin non engorgé, est faible ( $\leqslant 3$ bars), $50 \%$ des plants survivront, alors que quand il est supérieur à 3 bars, tous les plants concernés sont destinés à mourir à plus ou moins longue échéance.

Lors du ressuyage, le déficit en eau des plants destinés à survivre devient très rapidement inférieur à celui des plants encore engorgés; leurs racines tirent donc immédiatement parti du meilleur approvisionnement du milieu en oxygène.

Par contre, la pression de sève des plants destinés à mourir augmente beaucoup plus vite si lon ressuie ces plants que si on les laisse engorgés. Nous pressentions ce résultat, que nous pensons original, après les observations effectuées dans des expériences précédentes (LEVY, 1981 a) : le ressuyage de plants destinés à mourir accélérait très fortement le flétrissement des parties aériennes par rapport à des plants comparables maintenus engorgés (ces derniers dépérissaient cependant aussi, mais dans un délai plus long). Les racines des plants destinés à mourir ne profitent donc pas, au contraire, du meilleur approvisionnement du milieu en $O_{.2}$ dû au ressuyage; 
on peut penser qu’il n’y a plus (ou seulement très peu) d'alimentation en eau active (c'est-à-dire avec dépense d'énergie) possible; ces mêmes plants, s“ils demeurent au contraire engorgés, s'alimentent encore en eau, mais il s'agit sans doute d'un phénomène essentiellement passif, qui n'est possible qu'en présence d'une nappe libre (potentiel $\mathrm{O}$ ) et non si le sol est à la capacité au champ.

L'évolution quantitative des pressions de sève après ressuyage aurait vraisemblablement été modifiée si on avait effectué celui-ci plus progressivement que dans nos expériences, par exemple s"il avait duré plusieurs jours. Nous pensons cependant que le type d`évolution par rapport aux plants laissés engorgés n’aurait pas changé.

On suppose donc qu'il y a mortalité (à terme) des plants lorsque leurs racines ne sont plus en mesure de s'alimenter activement en eau, même en présence d'oxygène. Un maintien artificiel (par nébulisation) d'une teneur importante en eau dans les feuilles permet dailleurs de surseoir à leur mort.

\section{2. - Déficit hydrique diurne}

Dans le cas d'une nappe superficielle stagnante, un déficit d'alimentation en eau par rapport au témoin apparaît plus précocement de jour que de nuit, surtout en jour ensoleillé. Il est d’ailleurs normal que le déficit, pour des plants qui salimentent mal en eau, soit dautant plus important que la demande transpiratoire (ETP) est plus élevée. D'ailleurs, les besoins des racines en oxygène nécessaire notamment à l'alimentation en éau s'accroissent lorsque la température s'élève; ils sont done alors relativement plus mal satisfaits. élevées.

Ce déficit concerne rapidement tous les plants, et il atteint vite des valeurs

Le phénomène présente la même allure, mais son apparition est plus tardive, si la nappe est «oxygénée» ou si elle naaffleure quà - $3 \mathrm{~cm}$.

Par contre, dans le cas dune nappe à $-6 \mathrm{~cm}$, le déficit natteint finalement qu'une partie des plants (de 10 à $50 \%$ dentre eux en jours couverts, $75 \%$ en jours ensoleillés), et il ne dépasse jamais 7 bars.

La mortalité des plants n’apparaît que lorsque le déficit hydrique diurne est tel qu une réhydratation nocturne suffisante n’est plus possible.

\section{3. - Influence de l'état nutritif des plants engorgés sur lear alimentation en eau}

Des déficits minéraux internes importants contribuent à laugmentation ou même parfois à lapparition de déficits de lalimentation hydrique chez de jeunes plants d'Epicéa. Ainsi, une fertilisation préalable en $\mathrm{N}$ diminue beaucoup l'amplitude de ce déficit dans le cas d'une nappe superficielle, et peut même le supprimer totalement si la nappe est à $-6 \mathrm{~cm}$ (journées ensoleillées). 
Par ailleurs, une fertilisation préalable en $P$ a également un certain effet, mais nettement moins important; cet effet est favorable en sol pauvre en cet élément, défavorable en sol bien pourvu en $P$.

Les résultats concernant l'azote s'expliquent bien. En effet, une nappe superficielle supprime toute assimilation d'azote, alors que lorsque la nappe est située à - $6 \mathrm{~cm}$, deux comportements sont possibles : comme nous lavons montré dans un article précédent, certains plants assimilent l'azote, d'autres sont totalement carencés (Levy, 1981 b). Un parallèle simpose avec le fait signalé au paragraphe 3.23 : dans le cas d'une nappe à $-6 \mathrm{~cm}$, certains plants présentent un déficit hydrique diurne, tandis que dautres salimentent normalement en eau. Cette relation entre déficit hydrique et carence azotée n'est pas fortuite : on sait qu'un déficit en azote ainsi que lanoxie au niveau des racines augmentent la résistance des tissus racinaires au passage de l'eau, ces deux traitements renforçant mutuellement leurs effets lorsquils existent simultanément (Mingeau et Robel.iN, 1972; Morizitr, 1975).

En ce qui concerne l'effet favorable d’un apport de $P$ sur le déficit d’alimentation en eau, plusicurs hypothèses pourraient être avancées, par exemple que cet élément est nécessaire à l'absorption, ou au transfert, actifs de l'eau, car il contribue à la fourniture d'énergie à la racine par l"intermédiaire des réactions de phosphorylation. Daailleurs Morrzet et Mingeau (1976) ont montré que la carence en P, comme celle en $\mathrm{N}$, augmente les résistances à l'absorption de l'eau, bien que les symptômes soient plus longs à apparaître dans le cas de P. Quant à l'effet néfaste d'un apport de phosphore dans le cas d'un sol bien pourvu en cet élément, il est peut-être dû au fait qu'il provoque dans des plants très carencés en azote un trop important déséquilibre interne $d u$ rapport $\mathrm{N} / \mathrm{P}$, rapport dont on connait, sans $\mathrm{cn}$ comprendre la raison exacte, l'importance chez les résineux.

Rappelons que l'ensemble de ces résultats ne concerne que de jeunes plants engorgés laannće même de leur repiquage.

Reşu pour publication en janvier 1980.

\begin{abstract}
Summary
Influence of soil water logging conditions on water status evolution of young plants of Norway Spruce.
\end{abstract}

Young plants of Spruce were kept under different water logging conditions : surface or different depths water-table, oxygenated (air bubbling or daily water change) or not. Water status in plants was estimated by sap pressure, in no transpirating conditions (basic pressure) and with transpiration.

The main results are :

- Plant basic pressure (night measurements) decreases during the first days of water logging by a surface, non-oxygenated, water-table; after the 13th day, this pressure quickly increases. If the water-table is removed when basic pressure is at least 3 bars above the pressure of non-water-logged plants, all the plants die; water-table removal accelerates water deficit increase and the mortality of these plants, a lot.

With oxygenated surface water-table or $3 \mathrm{~cm}$ deep water-table, basic water deficit takes place later or never. 
- Water deficits take place first during sunny days, later if sky is overcast, and finally during night. In some treatments (deeper water-table, for instance $6 \mathrm{~cm}$ deep), water deficit only appears during day.

- Fertilizers addition have shown that nitrogen deficiency induced by water-table contributes much to the beginning or the importance of water deficit. It is likewise, but in a smaller extend, if there is a soil phosphorus deficit.

\section{Références bibliographiques}

Aussenac G. et Granier A., 1978. Quelques résultats de cinétique journalière du potentiel de sève chez les arbres forestiers. Ann. Sci. for., 35 (1), 19-32.

Barker J.E., 1973. Diurnal pattern of water potential in Abies concolor and Pinus resinosa. Can. J. For. Res., 3, 556-564.

BARRS H.D., 1965. Comparison of water potentials in leaves as Measured by two types of Thermocouple psychrometer. Aust. J. Biol. Sci., 18, 36-52.

Boyer J.S., 1967. Leaf water potentials measured with a pressure chamber. Plant Physiol., 42, $133-137$.

Boyer J.S., 1969. Measurement of the water status of plants. Ann. Rev. Pl. Physiol., 19, 351-363.

Brown R.W., 1976. New technique for measuring the water potential of detached leaf samples. Agron. J., 68, 432-434.

Heller R., 1969. Biologie végétale. II. Nutrition et métabolisme. Masson et Cie, Paris, $578 \mathrm{p}$.

Hinckliy T.M. et Ritchie G.A., 1973. A theoretical model for calculation of xylem sap pressure from climatological data. The American Midland Naturalist, 90 (1), 56-69.

Kaufmann M.R., 1968. Evaluation of the pressure chamber technique for estimating plant water potential of forest tree species. For. Sci., 14, 369-374.

Levy G., 1981, a). Comportement de jeunes plants d'Epicéa commun en sol à engorgement temporaire de surface : influence de divers facteurs du milieu. Ann. Sci. for., 38 (1).

Levy G., 1981, b). La nutrition azotée de l'Epicéa en sol engorgé : étude expérimentale. Ann. Sci. for, 38 (2).

Mazliak P., 1974. Physiologie végétale. Nutrition et métabolisme. Hermann, Paris, 350 p.

Mingead M., Robelin M., 1972. Le transfert de l'eau dans la plante : action particulière de la nutrition azolée. Ann. agron., 23 (4), 419-427.

Morizet J., 1975. Etude des transferts de l'eau dans la plante. Action d'une alimentation carencée en azote sur l'évolution de la teneur en eau des différents organes du Tournesol (Helianthus annuus). C.R. Acad. Sc. Paris, t. 281, série D, 1593-1596.

Morizet J., Mingeau M., 1976. Influence des facteurs du milieu sur l'absorption hydrique. Etude effectuée sur tomate décapitée. 1. Facteurs nutritionnels. Ann. agron., 27 (2), 183-205.

Ritchie G.A., Hinckiey T.M., 1971. Evidence for error in pressure-bomb estimates of stem xylem potentials. Ecology, 52, 534-536.

Ritchie G.A., Hinckily T.M., 1975. The pressure chamber as an instrument for ecological research. In advances in ecological research. A. Macfadyen Ed., vol. 9, 165-254, Academic Press, London.

Scholander P.F., Hammel H.T., Bradstreet E.D., Hemmingsen E.A., 1965. Sap pressure in vascular plants. Science, 148 (3668), 339-346.

Talbot A.J.B., Tyrel M.T., Dainty J., 1975. Some notes concerning the measurement of water potentials of leaf tissues with specific reference to Tsuga canadensis and Picea abies. Can. J. Bot., 53 (8), 784-788. 